\title{
Comparison of pressure plate and force plate gait kinetics in sound Warmbloods at walk and trot
}

\author{
Maarten Oosterlinck $^{\mathrm{a}, *}$, Frederik Pille ${ }^{\mathrm{a}}$, Tsjester Huppes ${ }^{\mathrm{b}}$, Frank Gasthuys ${ }^{\mathrm{a}}$, Willem Back ${ }^{\mathrm{a}, \mathrm{b}}$ \\ a Department of Surgery and Anaesthesiology, Faculty of Veterinary Medicine, Ghent University, B-9820 Merelbeke, Belgium \\ ${ }^{\mathrm{b}}$ Department of Equine Sciences, Faculty of Veterinary Medicine, Utrecht University, NL-3584 CM Utrecht, The Netherlands
}

\section{A R T I C L E I N F O}

\section{Article history:}

Accepted 18 August 2009

Available online $\mathrm{xxxx}$

\section{Keywords:}

Horse

Force plate

Gait analysis

Pressure plate

Symmetry

\begin{abstract}
A B S T R A C T
Modern pressure plates (PP) could be an alternative to traditional force plates (FP) for quantitative equine gait analysis, thereby providing the clinician with objective data on the horse's gait while unravelling the loading of different regions of the hoof during the stance phase. The aim of this study was to determine whether a stand-alone PP allows reliable measurement of gait kinetics, compared to simultaneously recorded FP variables. Six sound Warmblood horses were walked and trotted over a combined PP and FP system for collection of a set of five valid kinetic measurements for each forelimb. A measurement was considered valid if the horse was moving in a straight line at a constant pace while gait velocity was within a preset range and the hoof fully contacted the plate surface.

Significant differences between FP and PP data were seen for peak vertical force (PVF), vertical impulse (VI), time at which the PVF occurs (tPVF) and forelimb symmetry ratios (SymPVF and SymVI) $(P<0.05)$, but not for stance phase duration (ST). Nevertheless, mean agreement indices (AIs) of ST, tPVF and SymPVF and SymVI were excellent $(\geqslant 0.92)$, whereas AIs of PVF and VI were moderate $(\geqslant 0.70)$. The excellent agreement between PP and FP symmetry ratios confirms that observed differences between PP and FP in symmetry ratios are small (2-7\%), especially when compared to the expected decrease in symmetry associated with mild lameness ( $>20 \%$ ). The results indicate that a stand-alone pressure plate can be used to measure absolute (ST) and relative (tPVF) temporal variables and loading symmetry ratios and offers equine veterinarians a mobile, cost-efficient and quick gait evaluation method for routine clinical use. However, the system cannot be used interchangeably with a force plate to measure absolute values of limb loading.
\end{abstract}

(c) 2009 Elsevier Ltd. All rights reserved.

\section{Introduction}

Force plates (FPs) are accepted kinetic instruments for the quantitative evaluation of equine lameness (Buchner, 2001; Bertone, 2003; Ishihara et al., 2009). Despite their recognised accuracy, the use of FP systems is usually restricted to gait analysis under laboratory environments as installation procedures are laborious and costly as the FP requires a massive concrete foundation, while concurrent hoof strikes cannot be separated and limited plate dimensions preclude recording consecutive strides (Buchner, 2001; Bertone, 2003).

Modern portable pressure plate (PP) systems open new possibilities, whereby additional information on the pressure distribution of the different regions of the hoof during a complete stance period can be obtained (Van der Tol et al., 2003; Van Heel et al., 2004). This may allow us to differentiate toe and heel landing in specific causes of lameness (e.g. navicular syndrome vs. laminitis).

\footnotetext{
* Corresponding author. Tel.: +32 926476 18; fax: +32 2647794

E-mail address: Maarten.Oosterlinck@ugent.be (M. Oosterlinck).
}

PP measurements can be dynamically calibrated using simultaneous FP measurements (Van der Tol et al., 2003; Van Heel et al., 2004; De Cock et al., 2005; Hagman et al., 2008). However, the combination of a built-in and fixed FP and PP is really only feasibly in an institute or laboratory and is not practical use in a cost-efficient, mobile clinical environment. Solitary PPs, operational without concurrent FP calibration, have been applied in horses (Rogers and Back, 2007; Oosterlinck et al., 2009) and humans (Thijs et al., 2007). However, before kinetic data obtained with a standalone PP can be used clinically, validation of the system for precision (i.e. agreement between repeated measurements) and accuracy (i.e. agreement with reference standard) is necessary (Bossuyt et al., 2003).

A high level of precision of gait kinetics and loading symmetry ratios has been demonstrated in ponies using a stand-alone PP (Oosterlinck et al., 2009). Studies specifically dealing with the accuracy of a pressure measuring system compared to a FP have been undertaken in humans (Chesnin et al., 2000) and dogs (Besançon et al., 2003). The former reported excellent correlation between the centre of pressure determined by an in-shoe pressure 
measuring system and a FP, while the latter reported statistically significant differences in gait kinetics between the FP and PP systems. Equine bodyweight (BW), gait variables (stride length, etc.) and conformation are very different compared to other animal species and humans, necessitating direct comparison of simultaneously recorded PP and FP measurements.

A practical application can be foreseen when variables determined from the stand-alone PP could be used interchangeably with FP measurements, thereby providing valuable and objective information on the horse's gait and symmetry. This would offer the equine clinician a cost-efficient, quick and even portable gait evaluation method for routine clinical use. The objective of the present study was to compare gait kinetics in a group of sound horses using simultaneous measurements with a PP and a FP. The hypothesis was tested that the kinetic gait variables of the PP and the FP can be used interchangeably.

\section{Materials and methods}

\section{Horses}

Six healthy and clinically sound Dutch Warmblood mares were included in the study (mean age $7 \pm 4$ SD years, BW $592 \pm 82 \mathrm{~kg}$; wither height $1.59 \pm 0.07 \mathrm{~m}$ ). All horses were part of the teaching herd of Utrecht University, and were regularly used for pleasure riding by the student riding school. Prior to the study, each horse was physically evaluated by a veterinarian and judged to be healthy and sound.

The study was approved by the Ethical Committee of Utrecht University (2008.III.07.071).

\section{Measurement systems and data collection}

After a 5 min warm-up period, the horses were led over the measuring system by one experienced handler, first at the walk and subsequently at trot. The measuring system consisted of the PP (Footscan 3D 1 m-system, RsScan International) mounted on top of the force platform (Z4852C, Kistler) (Fig. 1), embedded flush and in the middle of a $20 \mathrm{~m}$ long track, covered with a $5 \mathrm{~mm}$ rubber mat. This setting has been used in equine (Van Heel et al., 2004), human (De Cock et al., 2005; Hagman et al., 2008) and bovine studies (Van der Tol et al., 2003). FP and PP data were collected simultaneously and recorded on separate notebook computers (Precision M6400, Dell) with dedicated software (Bioware 4, Kistler; Footscan Scientific Gait 7, RsScan International).

Calibration of the FP was verified before and shortly after the entire study. At the start of the study, the PP was calibrated in accordance with manufacturer's specifications using the RsScan software (Footscan Scientific Gait 7, RsScan International) with a person weighing $80 \mathrm{~kg}$. In an attempt to further optimise the calibration procedure, the recalibration factor obtained in this way was fine-tuned using one of the horses in the study. The horse was weighed with one forelimb standing on a calibrated weighing scale that was embedded flush in a concrete floor, standing squarely, while the contra-lateral forelimb was held in a flexed position without supporting weight. After the horse was placed on the PP in the same position, the constant vertical force recorded at that moment was compared with the previously recorded weight. The recalibration factor was changed manually until these two values matched for five consecutive calibration trials (i.e. a maximal difference of $5 \% \mathrm{BW})$.

The FP and the PP were both set at their routinely used measuring frequency ( $1000 \mathrm{~Hz}$ and $250 \mathrm{~Hz}$, respectively) so that for each frame of the PP, four FP frames were recorded. Both plates were triggered simultaneously by a photocell-activated gate $0.20 \mathrm{~m}$ in front of the measuring surface.

The average velocity over the measuring area was computed using two pairs of photoelectric-sensors (WE260-S270, Sick) that were placed as two gates perpendicular to the runway at $2 \mathrm{~m}$ interval centred over the measuring area, connected to an electronic timing box (Timer Interval Meter K3HB-P, Omron).

Although acceleration was not measured, the $20 \mathrm{~m}$ long track ensured that the effect of acceleration and deceleration at the start and end of each trial was minimised over the central $2 \mathrm{~m}$ measuring area. A trial was considered valid if the horse moved at a constant pace, looking straight forward, while gait velocity was within a preset range of $0.9-1.5 \mathrm{~m} / \mathrm{s}$ at the walk and $1.8-3.5 \mathrm{~m} / \mathrm{s}$ at the trot and the hoof of at least one forelimb fully contacted the plate surface. A total number of five valid measurements were collected for both forelimbs.

For both forelimbs of all horses, the following kinetic variables were calculated: (1) peak vertical force (PVF) was normalised by horses' bodyweight and expressed as Newtons per kilogram (N/kg); (2) vertical impulse (VI) was calculated by time integration of the force-time curves and multiplied by time, normalised by weight and expressed as Newton-seconds per kilogram ( $\mathrm{N} \mathrm{s} / \mathrm{kg}$ ); (3) stance time (ST) was calculated as the difference between the end and the beginning of the stance phase and was expressed in milliseconds (ms), and (4) time at which the PVF occurs (tPVF) was expressed as a percentile of the duration of stance time (\%stance).

\section{Data processing}

Only the data from the five valid trials at walk and at trot were analysed. For each set of five trials, the mean left fore (LF) and right fore (RF) value were calculated as being representative for that limb of each animal. Forelimb symmetry ratios (SymPVF and SymVI; \%) were calculated as lowest/highest mean value $\times 100 \%$, as described by Merkens et al. (1986).

\section{Statistical analysis}

Data were collated and prepared for statistical analysis using spreadsheet software (Microsoft Office Excel 2003) and statistical analysis was performed using SPSS 16.0 with statistical significance set at $P<0.05$. Data are presented as means $\pm \mathrm{SD}$, unless otherwise stated.

The assumption of normal distribution of all variables was confirmed using rankits plot, Kolmogorov-Smirnov and Shapiro-Wilk test. A linear Mixed Effects Model with horse as random component was used to evaluate (1) the effect of gait, LF or RF and measuring system on PVF, VI, ST and tPVF and (2) the influence of gait and measuring system on PVF and VI symmetry ratios.

To examine the agreement between PP and FP data, mean \pm SD agreement indices $(\mathrm{AI})$ were calculated for all variables. The following AI equation was used (Van der Vlugt-Meijer et al., 2006; White et al., 2008):

$\mathrm{AI}=1-[|\mathrm{PP}-\mathrm{FP}| /\{(\mathrm{PP}+\mathrm{FP}) / 2\}]$

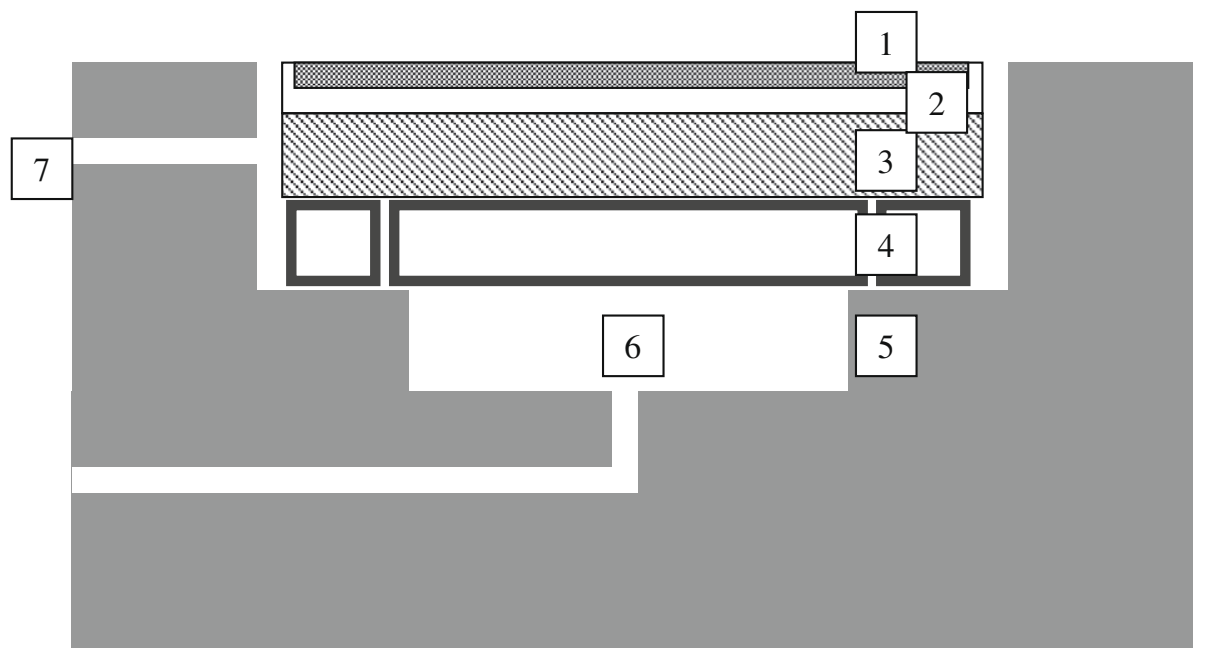

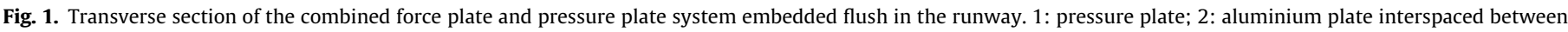
pressure plate and force plate; 3: force plate; 4: steel mounting frame of the force plate; 5: concrete foundation; 6: drain; 7: cable channel. 
An AI of 1 indicated perfect agreement and mean AIs $\geqslant 0.90$ represented excellent agreement (Van der Vlugt-Meijer et al., 2006; White et al., 2008), while $(0.7 \leqslant \mathrm{AI}<0.8)$ was considered moderate and $(0.8 \leqslant \mathrm{AI}<0.9)$ good agreement.

\section{Results}

A representative example of vertical force curves at walk and at trot, obtained with both measuring systems, is shown in Fig. 2. PP and FP measurements at walk and trot of all horses $(n=6)$, and AIs are summarised in Table 1.

Except for the symmetry ratios, analyses were conducted on pooled LF and RF data of the six horses since no significant differences were found between both forelimbs. All variables showed significant differences between the walk and trot. PVF, VI and tPVF obtained with the PP system were significantly different from the ones obtained from the FP, whereas only ST was not significantly different between the two measuring systems (Table 1). At walk and trot, SymPVF and SymVI of both systems were around $90 \%$, but the PP SymPVF and SymVI were nevertheless significantly lower than the FP values.

A significant interaction between gait and measuring system was seen for PVF and tPVF, but not for the other variables. Pairwise comparisons (Scheffé test) of combinations of interacting factors as separate groups (walk-PP, walk-FP, trot-PP and trot-FP) showed significant differences in all of these groups: at walk PP PVF was on average $1.4 \mathrm{~N} / \mathrm{kg}$ lower than FP PVF, while at the trot the mean difference was $2.7 \mathrm{~N} / \mathrm{kg}$. At walk, PP tPVF occurred on average 3.8\%
Table 1

Means \pm SD of concurrent pressure plate (PP, index test) and force plate (FP, reference standard) measurements at walk and trot in six horses with their agreement indices (AI).

\begin{tabular}{lccl}
\hline Variables & PP & FP & AI \\
\hline Walk & & & \\
PVF (N/kg) & $5.1 \pm 1.1^{\mathrm{a}}$ & $6.5 \pm 0.1^{\mathrm{b}}$ & $0.74 \pm 0.23$ \\
VI (Ns/kg) & $2.9 \pm 0.7^{\mathrm{a}}$ & $3.6 \pm 0.2^{\mathrm{b}}$ & $0.75 \pm 0.24$ \\
TPVF (\%stance) & $65.6 \pm 3.9^{\mathrm{a}}$ & $61.8 \pm 3.5^{\mathrm{b}}$ & $0.99 \pm 0.01^{\mathrm{c}}$ \\
ST (ms) & $834 \pm 38$ & $834 \pm 39$ & $1.00 \pm 0.00^{\mathrm{c}}$ \\
SymPVF (\%) & $93.7 \pm 3.2^{\mathrm{a}}$ & $98.2 \pm 0.9^{\mathrm{b}}$ & $0.95 \pm 0.04^{\mathrm{c}}$ \\
SymVI (\%) & $88.7 \pm 7.3^{\mathrm{a}}$ & $96.0 \pm 2.0^{\mathrm{b}}$ & $0.92 \pm 0.06^{\mathrm{c}}$ \\
Trot & & & \\
PVF (N/kg) & $7.8 \pm 1.5^{\mathrm{a}}$ & $10.4 \pm 0.7^{\mathrm{b}}$ & $0.69 \pm 0.19$ \\
VI (Ns/kg) & $1.5 \pm 0.4^{\mathrm{a}}$ & $2.0 \pm 0.2^{\mathrm{b}}$ & $0.72 \pm 0.20$ \\
tPVF (\%stance) & $51.9 \pm 4.6^{\mathrm{a}}$ & $43.0 \pm 2.9^{\mathrm{b}}$ & $0.95 \pm 0.01^{\mathrm{c}}$ \\
ST (ms) & $332 \pm 23$ & $333 \pm 23$ & $0.98 \pm 0.01^{\mathrm{c}}$ \\
SymPVF (\%) & $92.0 \pm 3.3^{\mathrm{a}}$ & $97.5 \pm 2.9^{\mathrm{b}}$ & $0.94 \pm 0.03^{\mathrm{c}}$ \\
SymVI (\%) & $92.0 \pm 4.0^{\mathrm{a}}$ & $94.8 \pm 2.4^{\mathrm{b}}$ & $0.97 \pm 0.05^{\mathrm{c}}$ \\
\hline
\end{tabular}

PVF, peak vertical force; tPVF, the time at which the PVF occurs; ST, stance phase duration; VI, vertical impulse; SymPVF and SymVI, forelimb symmetry ratios.

$\mathrm{a}, \mathrm{b}$ Within the same row, values with different lowercase superscript letters differ significantly $(P<0.05)$.

c Mean $A I \geqslant 0.90$ representing excellent agreement.

later in stance than in the FP measurements, while at trot it was on average $8.9 \%$ later.

Irrespective of gait, mean AIs for ST, tPVF, SymPVF and SymVI were $\geqslant 0.92$ with $\mathrm{SD} \leqslant 0.06$, while AIs for PVF and VI at both gaits were $\geqslant 0.70$ with $\mathrm{SD} \leqslant 0.24$ (Table 1 ).
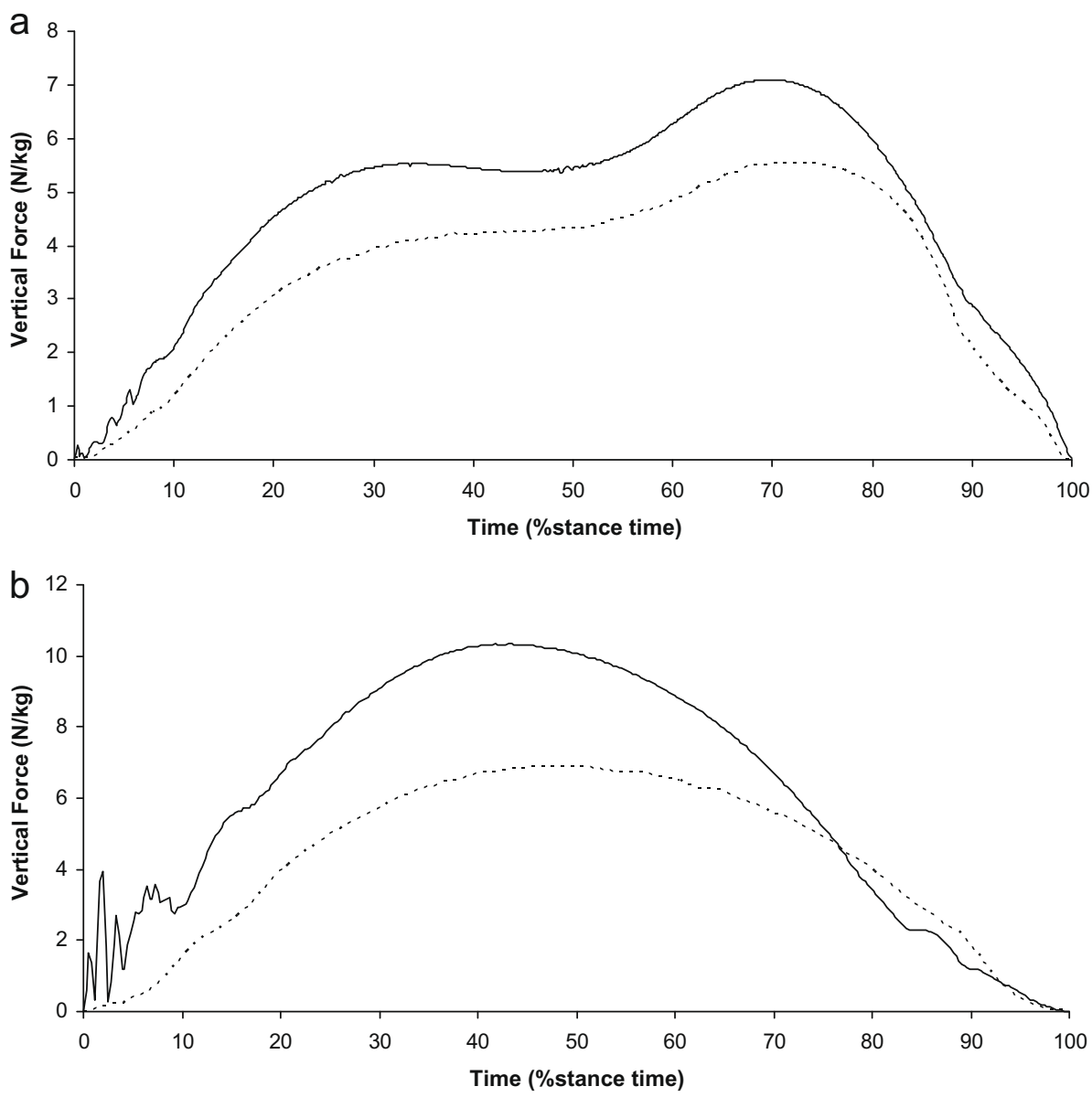

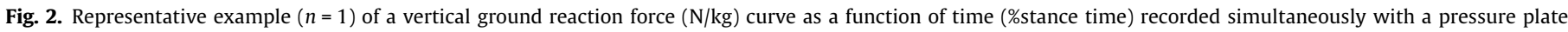
(dashed line) and a force plate (solid line) at walk (a) and trot (b). 


\section{Discussion}

The vertical force curves at walk and trot, obtained with both measuring systems, showed a similar pattern as reported earlier by Oosterlinck et al. (2009). Nevertheless, in the present study the actual PVF recorded with the PP showed a lower maximal value and was recorded somewhat later in the stance phase compared to the FP measurement.

Absolute stance duration recorded with PP and FP was similar and excellent agreement between both measuring systems was identified for relative temporal variables (tPVF). However, these temporal variables may not be useful kinetic parameters with which to predict equine gait anomalies, due to the rather large inter-individual variability and low sensitivity for detection of mild lameness (Ishihara et al., 2005). The observed differences in PVF and VI symmetry ratios (2-7\%; Table 1 ) were relatively small, whilst excellent agreement between PP and FP symmetry ratios was identified. Only moderate agreement was seen for absolute PVF and VI values.

A number of causes may explain the differences in data collected from the PP and the FP. There may be possible latency, and the temporal resolution of the sensors of the PP. PP PVF and VI were consistently lower than FP values. In contrast, a higher tPVF value was found for the PP compared to the FP whereas stance duration was highly similar between both systems. These findings might indicate a slower response (i.e. latency) of the multitude of resistor-based sensors of the PP compared to the four piezoelectric quartz crystal sensors of the FP. In the lower range of measurements, i.e. at the beginning and end of stance, the PP showed excellent alignment with the FP. Hagman et al. (2008) found no delays and no significant inaccuracies in the internal clocks of both systems and concluded synchronisation of the beginning and end of stance between both systems was excellent. However in the upper range of the forces in the present study, i.e. around PVF, the PP sensors showed a slower response than the FP. Moreover, at the trot PVF and tPVF showed larger differences than at walk thereby confirming larger deviations with increased loading.

The lower measuring frequency of the $\mathrm{PP}(250 \mathrm{~Hz})$ compared to the FP $(1000 \mathrm{~Hz})$ might also have contributed to the differences, although this would be likely to change the smoothness of the force-time curves rather than diminish the height of the curves. An illustration of this lower temporal resolution is the finding that the oscillations in the vertical force curve recorded with the FP at the beginning of the stance phase were smoothed in the PP curve (Fig. 2). The lower frequency of the PP may lead to missed data points and therefore a less accurate reading of the PVF, as suggested by Besançon et al. (2003) using another pressure measurement system in dogs. Nevertheless this is unlikely to be a major factor for the observed differences between PP and FP, as measuring frequency of the PP $(250 \mathrm{~Hz})$ was considered sufficiently high.

A further reason for differences between both measuring systems may be a suboptimal calibration of the PP. To the authors' knowledge, extensive data are lacking in the literature. Compared to the standard procedures specified by the manufacturer, Oosterlinck et al. (2009) used a refined calibration technique as described by Putti et al. (2008) whereby a static measurement was performed with a person standing on the PP on one limb. Subsequently, the recalibration factor was fine-tuned until the recorded vertical force was equivalent to the bodyweight of that person. As the linear response of the pressure-sensitive sensors may deviate under the heavy load of the adult Warmblood horses used in our study, it might be recommended to perform calibration in the same range as the actual measurements.

A static heavy weight in the range of a horse's BW is not readily available. Therefore our calibration procedure was refined using a horse standing with one forelimb on the plate. This can easily be performed in a practical setting. In the laboratory, PP measurements can be dynamically calibrated with a FP, as has been reported with humans (De Cock et al., 2005), horses (Van Heel et al., 2004) and cattle (Van der Tol et al., 2003). The presence of the PP on top of the FP does not change the vertical force signal in humans (Hagman et al., 2008), implying that continuous dynamic calibration of a PP using the vertical force signal of a FP is possible. However, this dynamic calibration was not done in the present study as we wanted to evaluate the clinical applicability of this portable PP as a stand-alone system.

The velocity range reported here $(0.9-1.5 \mathrm{~m} / \mathrm{s}$ at walk and $1.8-$ $3.5 \mathrm{~m} / \mathrm{s}$ at trot) was wider than that found in our previous study (1.1-1.5 and $2.5-3.5 \mathrm{~m} / \mathrm{s}$, respectively) to enhance simplicity of data collection. This undoubtedly caused larger inter-trial variability whereas in the previous work excellent repeatability of kinetic symmetry ratios was shown (Oosterlinck et al., 2009). However, in the present study variability was equivalent for the PP and the FP, as measurements with both systems were performed simultaneously. Although not considered in the present study, variability of gait kinetics can be a parameter of interest, as Ishihara et al. (2009) have shown that a unilateral decreased PVF and increased variability of gait kinetics may be considered as pathognomonic of musculoskeletal gait abnormality in horses.

Back et al. $(2007,2009)$ reported that visually scored grade $2 / 5$ lameness in Dutch Warmbloods is associated with a reduction of the absolute PVF from $118 \%$ to $89 \%$ BW, compatible with a decrease in symmetry around $20-25 \%$ or more, as compensatory overloading of the sound limb may occur. Therefore, the clinical relevance of the differences between the PVF and VI symmetry ratios in the present study appeared low, and the accuracy of the PP should be considered clinically acceptable for these symmetry ratios. This is in agreement with Besançon et al. (2003), who reported statistically significant differences in PVF and VI data between a FP and a pressure measuring system in dogs, but stated that the clinical relevance appeared negligible, indicating the pressure-measuring walkway was a viable alternative to the FP.

Although in the present study differences in symmetry ratios were observed between both measuring systems, PP kinetic symmetry ratios are highly repeatable (Oosterlinck et al., 2009) and this is critical in the objective evaluation of lameness resolution after local analgesia and longitudinal evaluation of orthopaedic treatments. Additionally, the use of a larger PP allows recording consecutive hoof strikes in one passage, at least in ponies (Oosterlinck et al., 2009). This is a powerful tool, as contralateral limbs are evaluated in one and the same passage, excluding inter-trial variability in the calculation of left-right symmetry. Besides these distinct advantages of this PP prospecting repeated gait assessments, pressure measuring equipment with high spatial and temporal resolution allows detailed evaluation of the pressure distribution within each hoof (Van der Tol et al., 2003; Van Heel et al., 2004).

\section{Conclusions}

We are aware that this stand-alone PP cannot simply replace a FP when high accuracy of absolute force values is needed for scientific purposes. Extended calibration may help to reduce measuring errors. Nevertheless, the available PP system can be used standalone to evaluate temporal variables and kinetic symmetry ratios in horses. Moreover, with PP measurements, the pressure distribution of the different regions of the hoof during a complete stance phase might become unravelled (Van der Tol et al., 2003; Van Heel et al., 2004), and analysis of simultaneous hoof impacts can be performed, which are not possible using a FP analysis. With these 
advantages, it is tempting to explore the use of the stand-alone PP to quantify equine lameness in a clinical setting. The present study opens perspectives to evaluate the use of the PP in sound and lame horses in order to demonstrate the utility of this pressure measuring system for repeated gait assessment in the horse (e.g. before and after application of diagnostic analgesia or corrective shoeing techniques).

\section{Conflict of interest statement}

None of the authors of this paper has a financial or personal relationship with other people or organisations that could inappropriately influence or bias the content of the paper.

\section{Acknowledgements}

The authors wish to thank Dr. Hans Vernooij (Utrecht University) and Professor Jeroen Dewulf (Ghent University) for their assistance with statistical analyses, and Dr. Meike Van Heel (Mustad Hoofcare) for critically reading the manuscript.

\section{References}

Back, W., MacAllister, C.G., van Heel, M.C., Pollmeier, M., Hanson, P.D., 2007. Vertical frontlimb ground reaction forces of sound and lame Warmbloods differ from those in Quarter Horses. Journal of Equine Veterinary Science 27, 123-129.

Back, W., MacAllister, C.G., van Heel, M.C., Pollmeier, M., Hanson, P.D., 2009. The use of force plate measurements to titrate the dosage of a new COX-2 inhibitor in lame horses. Equine Veterinary Journal 41, 309-312.

Bertone, A.L., 2003. Gait analysis for the quantification of lameness. In: Ross, M.W., Dyson, S.J. (Eds.), Diagnosis and Management of Lameness in the Horse. Saunders, St. Louis, USA, pp. 222-225.

Besançon, M.F., Conzemius, M.G., Derrick, T.R., Ritter, M.J., 2003. Comparison of vertical forces in normal greyhounds between force platform and pressure walkway measurement systems. Veterinary Comparative Orthopaedics and Traumatology 3, 153-157.

Bossuyt, P.M., Reitsma, J.B., Bruns, D.E., Gatsonis, C.A., Glasziou, P.P., Irwig, L.M., Moher, D., Rennie, D., de Vet, H.C.W., Lijmer, J.G., 2003. The STARD statement for reporting studies of diagnostic accuracy: explanation and elaboration. Croatian Medical Journal, 44: 639-650.

Buchner, H.H.F., 2001. Gait adaptation in lameness. In: Back, W., Clayton, H.M (Eds.), Equine Locomotion, first ed. W.B. Saunders, London, pp. 251-279.
Chesnin, K.J., Selby-Silverstein, L., Besser, M.P., 2000. Comparison of an in-shoe measurement device to a force plate: concurrent validity of center of pressure measurements. Gait and Posture 12, 128-133.

De Cock, A., De Clercq, D., Willems, T., Witvrouw, E., 2005. Temporal characteristics of foot roll-over during barefoot jogging: reference data for young adults. Gait and Posture 21, 432-439.

Hagman, F., Van de Ven, F., Van Gheluwe, B., Van Eijndhoven, S., 2008. Setting up gait analysis measurement devices for simultaneously measuring force, pressure, and motion. In: D'Août, K., Lescrenier, K., Van Gheluwe, B., De Clercq, D. (Eds.), Advances in Plantar Pressure Measurements in Clinical and Scientific Research. Shaker Publishing, Maastricht, pp. 26-43.

Ishihara, A., Bertone, A.L., Rajala-Schultz, P.J., 2005. Association between subjective lameness grade and kinetic gait parameters in horses with experimentally induced forelimb lameness. American Journal of Veterinary Research 66, 18051815.

Ishihara, A., Reed, S.M., Rajala-Schultz, P.J., Robertson, J.T., Bertone, A.L., 2009. Use of kinetic gait analysis for detection, quantification, and differentiation of hind limb lameness and spinal ataxia in horses. Journal of the American Veterinary Medical Association 234, 644-651.

Merkens, H.W., Schamhardt, H.C., Hartman, W., Kersjes, A.W., 1986. Ground reaction force patterns of Dutch warmblood horses at normal walk. Equine Veterinary Journal 18, 207-214.

Oosterlinck, M., Pille, F., Back, W., Dewulf, J., Gasthuys, F., 2009. Use of a standalone pressure plate for the objective evaluation of forelimb symmetry in sound ponies at walk and trot. The Veterinary Journal. doi:10.1016/ j.tvjl.2008.12.012.

Putti, A.B., Arnold, G.P., Cochrane, L.A., Abboud, R.J., 2008. Normal pressure values and repeatability of the Emed ST4 system. Gait and Posture 27, 501-505.

Rogers, C.W., Back, W., 2007. The effect of plain, eggbar and 6 degrees-wedge shoes on the distribution of pressure under the hoof of horses at the walk. New Zealand Veterinary Journal 55, 120-124.

Thijs, Y., Van Tiggelen, D., Roosen, P., De Clercq, D., Witvrouw, E., 2007. A prospective study on gait-related intrinsic risk factors for patellofemoral pain. Clinical Journal of Sport Medicine 17, 437-445

Van der Tol, P.P., Metz, J.H., Noordhuizen-Stassen, E.N., Back, W., Braam, C.R., Weijs, W.A., 2003. The vertical ground reaction force and the pressure distribution on the claws of dairy cows while walking on a flat substrate. Journal of Dairy Science 86, 2875-2883.

Van der Vlugt-Meijer, R.H., Meij, B.P., Voorhout, G., 2006. Intraobserver and interobserver agreement, reproducibility, and accuracy of computed tomographic measurements of pituitary gland dimensions in healthy dogs. American Journal of Veterinary Research 67, 1750-1755.

Van Heel, M.C., Barneveld, A., Van Weeren, P.R., Back, W., 2004. Dynamic pressure measurements for the detailed study of hoof balance: the effect of hoof trimming. Equine Veterinary Journal 36, 778-782.

White, J.M., Mellor, D.J., Duz, M., Lischer, C.J., Voute, L.C., 2008. Diagnostic accuracy of digital radiography and image analysis for the measurement of foot conformation in the horse. Equine Veterinary Journal 40, 623-628. 